\title{
Hybrid remote quantum dot/powder phosphor designs for display backlights
}

\begin{abstract}
Sofie Abe ${ }^{1,2,3}$, Jonas J Joos ${ }^{2,3}$, Lisa IDJ Martin ${ }^{2,3}$, Zeger Hens ${ }^{1,3}$ and Philippe F Smet ${ }^{2,3}$
Quantum dots are ideally suited for color conversion in light emitting diodes owing to their spectral tunability, high conversion efficiency and narrow emission bands. These properties are particularly important for display backlights; the highly saturated colors generated by quantum dots justify their higher production cost. Here, we demonstrate the benefits of a hybrid remote phosphor approach that combines a green-emitting europium-doped phosphor with red-emitting CdSe/CdS core/shell quantum dots. Different stacking geometries, including mixed and separate layers of both materials, are studied at the macroscopic and microscopic levels to identify the configuration that achieves maximum device efficiency while minimizing material usage. The influence of reabsorption, optical outcoupling and refractive index-matching between the layers is evaluated in detail with respect to device efficiency and cost. From the findings of this study, general guidelines are derived to optimize both the cost and efficiency of $\mathrm{CdSe} / \mathrm{CdS}$ and other (potentially cadmium-free) quantum dot systems. When reabsorption of the green and/or red emission is significant compared to the absorption strength for the blue emission of the pumping light emitting diode, the hybrid remote phosphor approach becomes beneficial.
\end{abstract}

Light: Science \& Applications (2017) 6, e16271; doi:10.1038/lsa.2016.271; published online 2 June 2017

Keywords: cost-efficiency; lanthanide; light emitting diode; nanocrystal; remote phosphor

\section{INTRODUCTION}

The image quality of displays has been substantially improved through the use of white-light emitting diodes (wLEDs) for backlighting. Current wLEDs are composed of a blue pump LED and one or more color convertors, often called phosphors. The emission spectrum of wLEDs can thus be adjusted based on the shape, peak wavelength, and relative intensities of the blue LED and color convertor emission ${ }^{1,2}$. Display applications require saturated colors; the optimal configuration demands a spectrum composed of primary colors (RGB) corresponding to monochromatic light with wavelengths of 630, 532, and $473 \mathrm{~nm}$, respectively ${ }^{3}$. Producing such monochromatic light from phosphor converted (pc) wLEDs based on broad-emitting lanthanide-doped materials is possible only through aggressive filtering. This, however, induces important output losses that make a tradeoff between color saturation and energy efficiency unavoidable.

Current developments in wLEDs for displays focus on narrowemitting color convertors, which can enhance a display's color gamut without sacrificing power efficiency. In this respect, red phosphors with a narrow emission band have been proposed, such as $\mathrm{K}_{2} \mathrm{SiF}_{6}: \mathrm{Mn}^{4+}$ or $\mathrm{SrLiAl}_{3} \mathrm{~N}_{4}: \mathrm{Eu}^{2+}$ (Refs 4,5). Moreover, new luminescent materials, such as colloidal quantum dots (QDs), have gained considerable attention $^{2,6-15}$. They combine a broad absorption spectrum with a tunable, spectrally narrow (full-width at half-maximum (FWHM) $<40 \mathrm{~nm}$ ) and efficient emission, which enables emission spectra to be adjusted for the needs of specific applications. In addition, their suitability for solution processing can reduce device fabrication cost and enhances freedom-of-design, for example, to make flexible displays ${ }^{16-20}$. Recent progress to improve their stability has made QDs competitive with common lanthanide-doped phosphors ${ }^{7,21,22}$. So far, the best results for pc wLEDs in commercial displays have been obtained using Cd-containing QDs since these show the highest conversion efficiency, narrowest emission, best stability, and greatest freedom to tune the absorption spectrum ${ }^{10,11,21-25}$. Nevertheless, efforts continue to be made to develop better-performing Cd-free $\mathrm{QDs}^{26}$, with the most promising being InP-based QDs ${ }^{10,19,26-31}$ and cesium lead halide perovskites ${ }^{32-34}$.

For commercial QD-based displays, designs in which the QDs are deposited on top or at the edge of the light guide plate are preferred over their direct on-chip deposition on the blue pump LED ${ }^{14}$. This so-called remote phosphor approach has several advantages, such as improved color mixing, efficiency, and photo and thermal stability ${ }^{10,26,35}$. On the other hand, it requires more phosphor material for the color conversion than on-chip designs. In addition, because QDs are too small to significantly scatter light, down-converted light can be trapped in the remote phosphor layer. The resulting outcoupling losses reduce the overall optical efficiency of the display ${ }^{14,36}$ and again increase the amount of phosphor material needed to balance the intensity of the down-converted luminescent emission to the transmitted pump light. These issues demand strategies that both

${ }^{1}$ Department of Inorganic and Physical Chemistry, Ghent University, Gent, Belgium; ${ }^{2}$ Department of Solid State Sciences, LumiLab, Ghent University, Gent, Belgium and ${ }^{3}$ Center for Nano and Biophotonics, Ghent University, Gent, Belgium

Correspondence: Z Hens, Email: zeger.hens@ugent.be; PF Smet, Email: philippe.smet@ugent.be

Received 18 July 2016; revised 5 December 2016; accepted 7 December 2016; accepted article preview online 9 December 2016 
enhance optical efficiency and reduce the use — and thus the cost—of QDs in color convertors for display applications.

Here, we present the concept of a hybrid remote phosphor layer, in which a lanthanide-doped powder phosphor (PP) is combined with QDs, as an approach to optimize the performance over cost ratio of color convertors for displays. On the basis of the premise that the strong scattering by microcrystals of the PP in such a hybrid system can enhance light outcoupling and thus the overall optical efficiency, we explore the interplay between both emitters using a combination of green-emitting microcrystalline PP, based on $\mathrm{SrGa}_{2} \mathrm{~S}_{4}: \mathrm{Eu}^{2+}$, and red emitting CdSe/CdS quantum dots. For this purpose, we compare different remote phosphor designs that consist of either a single, mixed layer or a stack of layers each containing only a single color convertor. Both materials are highly efficient color convertors, which enables us to express the cost vs performance tradeoff in terms of intrinsic characteristics of the color convertors. This approach can be readily extended to other hybrid $\mathrm{QD} / \mathrm{PP}$ combinations. In the first part of this work, the properties of the single and mixed color conversion layers are discussed. Then, we evaluate the stacking geometry, which is instrumental in assessing the influence of scattering and outcoupling on the spectral distribution and overall conversion efficiency. This knowledge makes it possible to optimize the loading of the individual layers in the different stacking geometries in order to attain the desired color point for display applications.

\section{MATERIALS AND METHODS}

\section{Color conversion materials}

CdSe wurtzite core QDs were synthesized according to a method adapted from the literature (see Supplementary Information) ${ }^{37}$. Their diameter and concentration were determined from the absorbance spectrum of a diluted suspension in chloroform using the peak wavelength of the first exciton transition $\left(\lambda_{\max }\right)$ and the absorbance at $350 \mathrm{~nm}$, respectively ${ }^{38}$. Red-emitting $\mathrm{CdSe} / \mathrm{CdS}$ core-shell structures were synthesized via a seeded growth procedure ${ }^{39}$. A greenemitting europium-doped strontium thiogallate (STG) phosphor based on $\mathrm{SrGa}_{2} \mathrm{~S}_{4}: \mathrm{Eu}^{2+}$ was used as the green component. Remote phosphor layers containing either STG powder, CdSe/CdS QDs, or a mixture of both were prepared by mixing appropriate amounts with a solution of 20 mass\% Kraton FG1901X (in 3:1 toluene) and 80 mass\% methyl ethyl ketone, stirring, and drop casting this mixture on a 0.2 -mm-thick circular glass substrate with a diameter of $18 \mathrm{~mm}$. After solvent evaporation, homogeneous color convertor layers with a thickness of $\sim 290 \mu \mathrm{m}$ were obtained (see Supplementary Information).

\section{Hybrid remote phosphor converted white LEDs}

Remote pc wLEDs were fabricated by combining these color convertor layers with a blue pump LED. The following geometries were studied: (1) a mixed, hybrid phosphor layer containing both materials, denoted as IRGI; (2) a stack of two layers separated by a 1-mm air gap, realized by using two small glass spacers $\left(1.5 \times 1.5 \mathrm{~mm}^{2}\right)$ and denoted as $|\mathrm{R} \| \mathrm{G}|$ (STG on top) or IGIIRI (QDs on top); and (3) similar stacks where the gap is filled with ethylene glycol for refractive index-matching, denoted as IR"IGI and IGHiRI, respectively.

\section{Optical characterization}

Optical characterization involving absorption and luminescence spectroscopy and luminescence lifetime measurements was performed as outlined in the Supplementary Information. The quantum efficiency (QE) of the unprocessed color convertors-phosphor powder and dispersed QDs-was determined using an integrating sphere.
The internal (IQE) and external (EQE) QE of the PP were determined via the two measurement approach ${ }^{40}$, in which the $\mathrm{EQE}$ is the ratio between the numbers of emitted and incident photons and the IQE equals the ratio between the numbers of emitted and absorbed photons. Typically, an uncertainty of $5 \%-10 \%$ is associated with the determination of QE values. The IQE of QDs was obtained by analyzing the absorption of blue pump light $(400-490 \mathrm{~nm})$ and red luminescence $(550-725 \mathrm{~nm})$ of toluene-based dispersions with an absorbance of $0.05-0.1$ in a $10 \mathrm{~mm}$ quartz cuvette, compared with a cuvette containing toluene only. Phosphor layers and wLED configurations were analyzed by introducing the circular layer(s) to a cylindrical, white Teflon mixing chamber with a height of $20 \mathrm{~mm}$, which contains a $453 \mathrm{~nm}$ high-power LED at the bottom for excitation (see Supplementary Information). The mixing chamber is inserted in the integrating sphere such that the top layer aligns with the inner surface of the sphere. QEs of distinct phosphor layers and hybrid wLED configurations, either mixed or a combination of two stacked layers on top of each other, were calculated relative to, respectively, one or two glass substrates. Simulations of phosphor combinations for wLEDs were performed using the NIST-CQS software (Physical Measurement Laboratory, Gaithersburg, MD, USA $)^{41-43}$.

\section{Structural characterization}

Samples for transmission electron microscopy were prepared by drop casting a dilute dispersion of QDs in toluene on a carbon-coated copper grid. Bright-field transmission electron microscopy images were recorded using a Cs corrected JEOL 2200 FS (JEOL, Tokyo, Japan) microscope. SEM-EDX-CL measurements were performed at room temperature using a Hitachi (Hitachi High-Technologies Co. Ltd., Tokyo, Japan) S-3400N scanning electron microscope (SEM), equipped with a Thermo Scientific (Thermo Fisher Scientific Inc., Waltham, MA, USA) Noran System 7 energy-dispersive X-ray detector (EDX) for chemical analysis. Cathodoluminescence (CL) was collected using an optical fiber ${ }^{44}$ and analyzed using the aforementioned monochromator and detector of the QE setup.

\section{RESULTS AND DISCUSSION}

\section{Single phosphor color conversion layers}

We form a hybrid remote QD/PP by combining wurtzite CdSe/CdS core/shell QDs, with $\lambda_{\max }$ at $628.5 \mathrm{~nm}$, an FWHM of $39.0 \mathrm{~nm}$ and an IQE of $79 \%$, as the red emitter with STG microcrystals as green phosphor with a $51.5-\mathrm{nm}$-wide emission band centered at $534.5 \mathrm{~nm}$ (Figure 1a). When the STG powder is measured in reflection mode, QEs amount to $95 \%$ (IQE) for an absorption of $80 \%$ of the incident blue light, leading to an EQE of $\sim 75 \%$. Thanks to the thick CdS shell $(\approx 3.3 \mathrm{~nm})$, the absorption spectrum of the QDs shows a marked increase at wavelengths shorter than $515 \mathrm{~nm}$, corresponding to the bulk bandgap of CdS (see Figure 1a). More quantitatively, the CdSe/ CdS QD absorption coefficient at $453.5 \mathrm{~nm}$ (pump wavelength) exceeds that at either $534.5 \mathrm{~nm}$ (STG luminescence) or $628.5 \mathrm{~nm}$ (QD luminescence) by a factor of 15 and 78, respectively. As this will translate into a proportionally longer path length for green and red light, we expect reabsorption-where a QD is excited by a red photon emitted by another QD or a green instead of a blue photon-to be relatively limited with these CdSe/CdS QDs.

The emission spectra of STG and CdSe/CdS fall primarily within the range of the respective green and red color filters currently used in displays (see Supplementary Information). The suitability of their combination as a remote phosphor for display applications is further confirmed by analyzing the spectrum of a hypothetical wLED fabricated with these building blocks in optimized quantities, which 

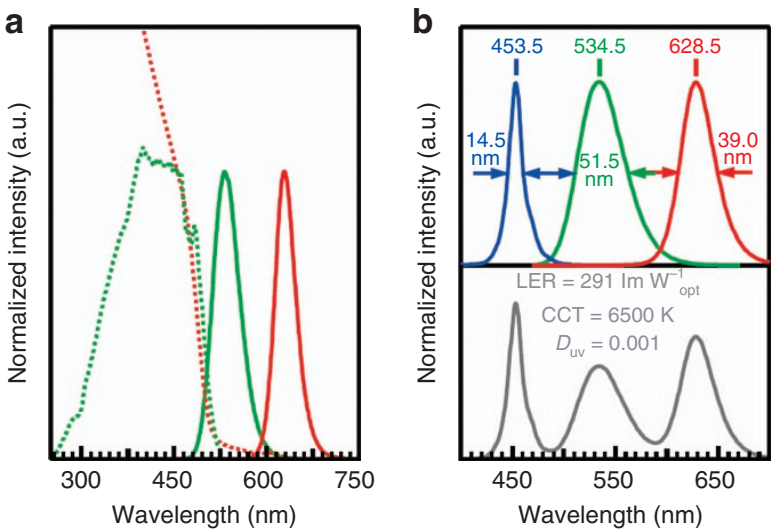

C

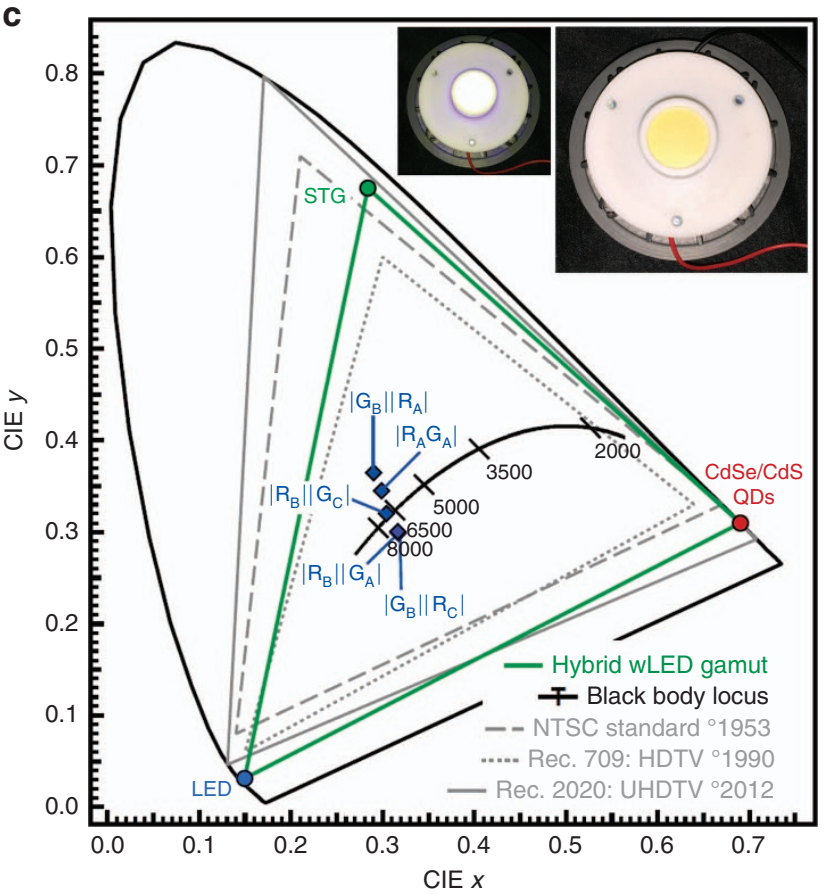

Figure 1 (a) Normalized excitation (green, dotted line) and emission (green, solid line) spectra of STG powder. Absorbance (red, dotted line) and emission (red, solid line) of a diluted dispersion of CdSe/CdS QDs. Emission spectra are obtained from excitation at $450 \mathrm{~nm}$. (b) Optimized white LED spectra from simulations in gray (maximal LER, $\left|D_{\text {uv }}\right|<0.001, C C T=6500 \mathrm{~K}$ ) composed of a Royal-Blue LUXEON Rebel ES LED (blue), STG phosphor (green), and CdSe/CdS QDs (red). (c) CIE 1931 chromaticity diagram with the color points of the used RGB components (circles) and hybrid wLEDs (blue diamonds) discussed in this paper, the black-body locus and the color gamut of specific display standards. The insets show the white reflective cup and the $\left|R_{A} G_{A}\right|$ layer with (left) and without (right) blue excitation.

features a luminous efficiency of radiation (LER) of $290 \mathrm{~lm} \mathrm{~W}^{-1}$ (Figure 1b). Moreover, the color points of the blue LED, the dilute dispersion of the QDs and the STG powder in the Commission internationale de l'éclairage (CIE) $x, y\left(u^{\prime}, v^{\prime}\right)$ color space already yield a color gamut covering $82 \%(86 \%)$ of the NTSC standard, before the application of a color filter (Figure 1c). Hence, we conclude that the combination of STG PP and CdSe/CdS QDs yields a state-of-the-art remote phosphor that can form an alternative to QD-only approaches and simultaneously offers an attractive starting point for analyzing the general properties of hybrid QD/PP remote phosphors. a

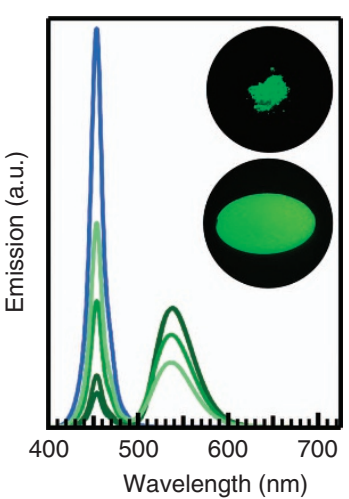

C

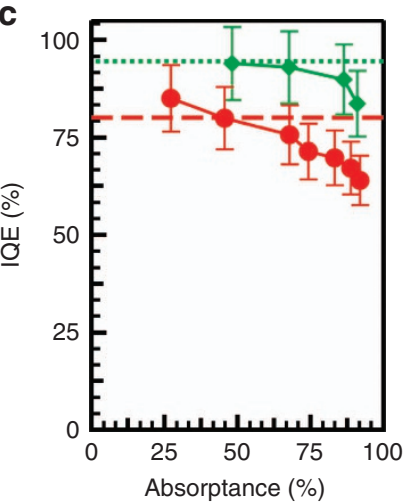

b

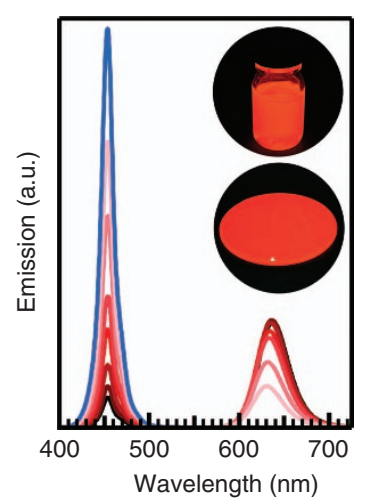

d

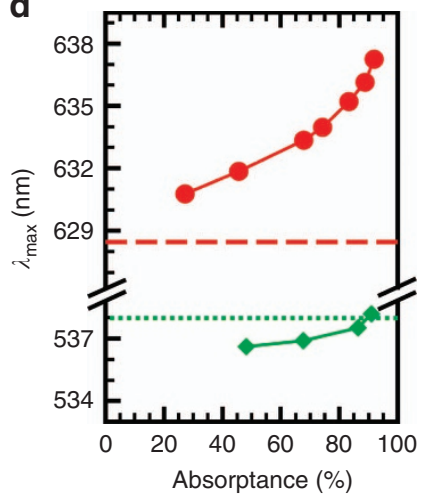

Figure 2 Emission spectra of the conversion layers, excited by the pump LED (blue), containing various amounts of (a) STG powder and (b) CdSe/CdS QDs. The insets show the raw material and the prepared layers under ultraviolet illumination. (c) Internal QE and (d) peak wavelength of phosphor layers with different absorption, containing either STG powder (green diamonds) or CdSe/CdS QDs (red circles).

Figure $2 \mathrm{a}$ and $2 \mathrm{~b}$ shows the emission spectra of various single phosphor layers containing either STG or QDs in different amounts, excited by a $453.5 \mathrm{~nm}$ LED and measured in a remote configuration in an integrating sphere. The absorbed fraction or absorptance $A$ is calculated as $1-T$, with $T$ being the transmittance of blue light through a color conversion layer. The latter is obtained from the ratio of the integrated intensity of blue light transmitted through the entire color convertor stack to that through the glass plate supporting the layers only. We thereby neglect any transmission loss from additional reflections other than that arising from the glass substrate. For each layer, $A$ is changed by incorporating different amounts of a color convertor, keeping the amount of binding polymer constant. Table 1 presents an overview of the composition and properties of the raw materials and single phosphor layers. To attain a similar absorptance, the weight of the PP is 20-fold that of the QDs.

Figure $2 \mathrm{c}$ indicates that an increasing absorptance reduces the IQE of the QD-based layers from $\sim 80 \%$ for QD dispersions with a $10 \%-20 \%$ absorptance down to $64 \%$ for a $92 \%$ absorptance layer. This concurs with a progressive redshift of the emission peak $\lambda_{\max }$ by $8.8 \mathrm{~nm}$ compared with $\lambda_{\max }$ of a QD dispersion (see Figure $2 \mathrm{~d}$ ). Both effects point to an enhanced self-absorption, where photon recycling lowers the overall IQE and shifts the emission spectrum to longer wavelengths, induced by light guiding in the remote phosphor film. In contrast, the emission of STG shows only very slight spectral shifts or efficiency loss upon embedding of the microcrystallites in the polymer 
Table 1 Overview of the composition and properties of the raw materials and single phosphor layers

\begin{tabular}{|c|c|c|c|c|c|c|c|c|}
\hline & Dilute QD dispersion & $\left|R_{A}\right|$ & $\left|R_{B}\right|$ & $\left|R_{C}\right|$ & $\mathrm{SrGa}_{2} \mathrm{~S}_{4}: \mathrm{Eu}^{2+}$ powder & $\left|G_{A}\right|$ & $\left|G_{B}\right|$ & $\left|G_{C}\right|$ \\
\hline QDs $\left(\mathrm{g} \mathrm{m}^{-2}\right)$ & - & 1.6 & 0.5 & 1.1 & - & - & - & - \\
\hline IQE (\%) & 79 & 71 & 80 & 76 & 95 & 93 & 94 & 94 \\
\hline$\lambda_{\max , \mathrm{em}}(\mathrm{nm})$ & 629 & 634 & 632 & 633 & 535 & 537 & 537 & 537 \\
\hline$\tau_{\mathrm{av}, \mathrm{em}}(\mathrm{ns})$ & 286 & 235 & - & - & - & 486 & - & - \\
\hline
\end{tabular}

a

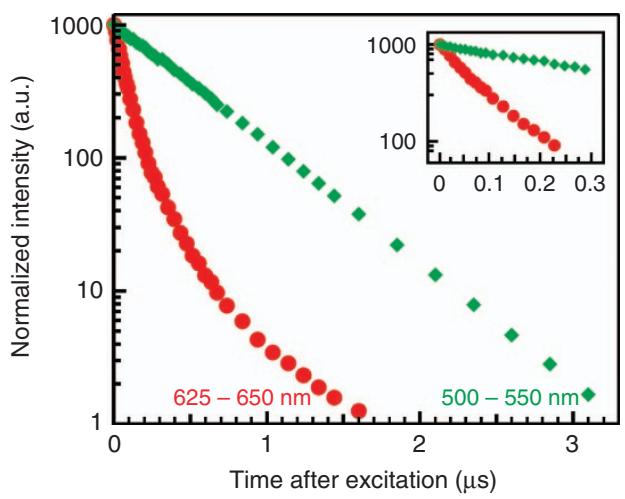

b

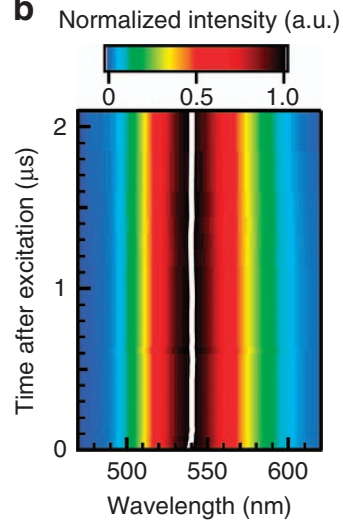

C

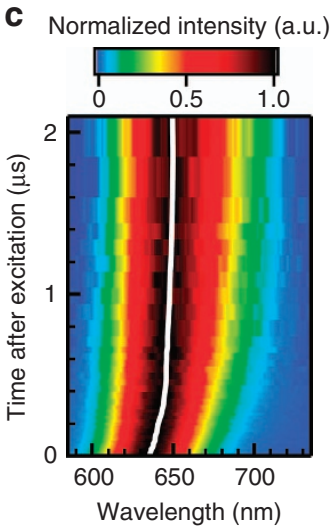

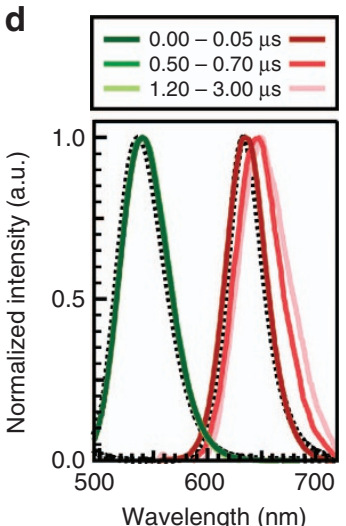

Figure 3 (a) Decay of the luminescence intensity of color-converting layers containing STG powder (IGAl, green diamonds) and CdSe/CdS QDs (IRAl, red circles), integrated from 500 to $550 \mathrm{~nm}$ and 625 to $650 \mathrm{~nm}$, respectively. (b, c) show the change of the normalized emission spectra of both layers with time, with the white line indicating $\lambda_{\max }$. (d) Emission spectra of $\left|G_{A}\right|$ (solid green lines) and $\left|R_{A}\right|$ (solid red lines) for different time intervals after excitation. The dotted lines represent the steady-state emission of the layers.

layer (Figure $2 \mathrm{c}$ and $2 \mathrm{~d}$ ), indicating that self-absorption is of little significance here. Hence we can use the steady-state spectral shift of the red emission peak as an indicator of reabsorption in the color conversion layers.

Figure 3a shows the time-resolved luminescence of $\left|G_{A}\right|$ (that is, a layer with $19.6 \mathrm{~g} \mathrm{~m}^{-2}$ of STG, see Table 1 ), which follows a single exponential decay with a lifetime $\tau=486 \mathrm{~ns}$. Moreover, as indicated by Figure $3 \mathrm{~b}$ and $3 \mathrm{~d}$, the emission spectrum does not alter with time, supporting the earlier conclusion that self-absorption is not important in these layers. The decay of the red emission band of $\left|R_{A}\right|-$ a layer containing $1.6 \mathrm{~g} \mathrm{~m}^{-2}$ of CdSe/CdS QDs with $A=74 \%$ - on the other hand is better modeled by a bi-exponential decay with an average decay constant $\tau_{\mathrm{av}}$ of $235 \mathrm{~ns}$ (see Supplementary Information for a detailed analysis). Moreover, Figure $3 \mathrm{c}$ and $3 \mathrm{~d}$ shows that the emission spectrum of $\left|R_{A}\right|$, measured in a remote phosphor configuration, changes throughout the decay. The peak wavelength $\lambda_{\max }$ shifts from $635 \mathrm{~nm}$ during the first $10 \mathrm{~ns}$ to $649 \mathrm{~nm}$ after $1.2 \mu \mathrm{s}$, while the FWHM increases from 43 to $55 \mathrm{~nm}$. This can be caused by a polydisperse CdS shell thickness as both the decay time and $\lambda_{\max }$ of the QDs increase with the CdS shell thickness, yet it can also reflect the self-absorption discussed above, which will redshift the PL. As the peak wavelength $\lambda_{\max }$ of the dilute dispersion redshifts by $9 \mathrm{~nm}$ through the decay, while the FWHM increases from 38 to $49 \mathrm{~nm}$, we conclude that both heterogeneity and self-absorption influence the emission properties of the QD layer.

\section{A mixed two-color QD/PP conversion layer}

Hybrid QD/PP layers can be fabricated by either embedding both emitters in a single IRGI layer or stacking two layers containing a single emitter each $22,35,43$. Either the QD or the STG layer can be closest to the blue LED, which are labeled $|R \| G|$ and $|G \| R|$, respectively. The emission spectrum of each configuration depends on the interplay between the relative absorption of the blue pump light by both color convertors, the scattering of light by the PP, and reabsorption of green and red emission by the $\mathrm{QDs}^{45}$. Because all these processes may depend on the design of the color conversion layers-mixed IRGI, stacked $|R \| G|$ or $|G||R|$ - we take the mixed IRGI films to benchmark the other geometries.

As shown in Figure $4 a$, a mixed $\left|R_{A} G_{A}\right|$ hybrid layer (see Table 2 for characteristics) yields, upon pumping with the blue pump LED, a correlated color temperature (CCT) of $7082 \mathrm{~K}$ and CIE $(x, y)$ color coordinates of $(0.299,0.345)$. Obviously, this color point can be brought closer to the black-body locus (Figure 1c) by slightly adjusting the relative loading in the film, yet this is not the main goal of this study. At $80 \%$, the IQE of the $\mid R_{A} G_{A} I$ film lies between the IQE of the individual $\left|R_{A}\right|(71 \%)$ and $\left|G_{A}\right|(93 \%)$ layers. Moreover, the 5-nm redshift of the QD emission's $\lambda_{\max }$ in the $\mid \mathrm{R}_{\mathrm{A}} \mathrm{l}$ layer relative to the $\mathrm{QD}$ dispersion is reduced to less than $1 \mathrm{~nm}$ in $\left|R_{A} G_{A}\right|$ (see Tables 1 and 2), substantiating a significant reduction of the QD self-absorption, due to the enhanced outcoupling of the red light through scattering by the STG microcrystals. When the degree of scattering is too high, multiple scattering events will increase the path length of the QD emission and thus also reabsorption, leading to efficiency losses and a redshifted emission. This is not the case in the present STG layers, as the scattering is just sufficient to redirect trapped (red) light that did not immediately leave the film. The scattering coefficient for $\left|G_{A}\right|$ (Table 1) was found to be $42 \mathrm{~cm}^{-1}$ (see Supplementary Information). Given that the degree of scattering depends on the size (and the size distribution), 
a

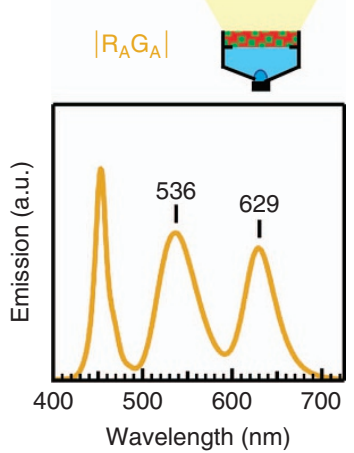

b

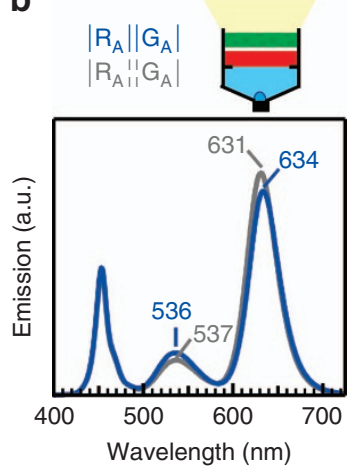

c

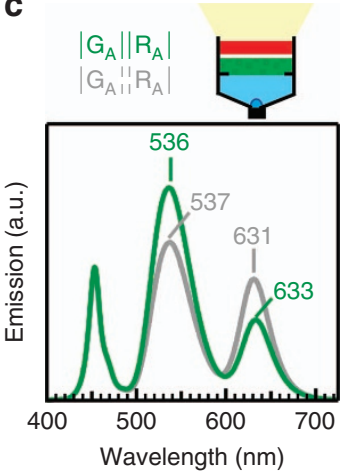

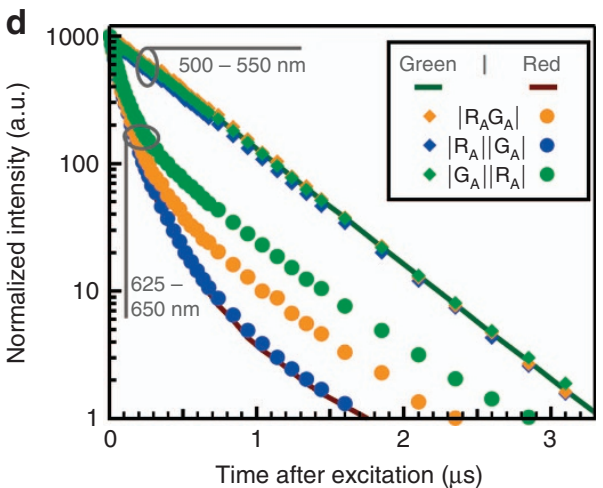

Figure 4 Emission spectra of a remote phosphor wLED composed of a blue pump LED in a white mixing chamber containing (a) a hybrid phosphor layer $\left|R_{A} G_{A}\right|$, (b) two stacked color-converting layers without ( $\left|R_{A} \| G_{A}\right|$, blue) and with ( $\left|R_{A} \|: G_{A}\right|$, dark gray) optical contact, and (c) the reversed configurations $\left|G_{A} \| R_{A}\right|$ (green) and $I G_{A} \| R_{A} I$ (light gray). The figures on the top are a schematic representation of each configuration. (d) Decay of the green and red luminescence intensities. The solid lines represent the decay of the color-converting layers, the symbols that of the hybrid wLED configurations.

Table 2 Overview of the composition and properties of remote hybrid wLEDs

\begin{tabular}{|c|c|c|c|c|c|c|c|c|c|}
\hline & $\left|R_{A} G_{A}\right|$ & $\left|R_{A} \| G_{A}\right|$ & $\left|G_{A} \| R_{A}\right|$ & $\left|R_{A} \| G_{A}\right|$ & $\left|G_{A} \| R_{A}\right|$ & $\left|R_{B} \| G_{C}\right|$ & $\left|G_{B} \| R_{A}\right|$ & $\left|R_{B} \| G_{A}\right|$ & $\left|G_{B}\right|\left|R_{C}\right|$ \\
\hline QDs $\left(\mathrm{g} \mathrm{m}^{-2}\right)$ & 1.6 & 1.6 & 1.6 & 1.6 & 1.6 & 0.5 & 1.6 & 0.5 & 1.1 \\
\hline$\lambda_{\max , \text { green }}(\mathrm{nm})$ & 537 & 536 & 536 & 537 & 537 & & 536 & 536 & 537 \\
\hline$\lambda_{\text {max,red }}(\mathrm{nm})$ & 630 & 634 & 633 & 631 & 631 & & 633 & 631 & 631 \\
\hline$\tau_{\mathrm{av}, \mathrm{red}}(\mathrm{ns})$ & 369 & 230 & 455 & 233 & 474 & - & - & - & - \\
\hline LE (Im W-1 electr) & 105 & - & - & - & 119 & 89 & 105 & 86 & 86 \\
\hline CIE $x$ & 0.299 & 0.416 & 0.287 & 0.416 & 0.318 & 0.304 & 0.290 & 0.316 & 0.317 \\
\hline CIE $y$ & 0.345 & 0.262 & 0.461 & 0.262 & 0.418 & 0.320 & 0.365 & 0.300 & 0.299 \\
\hline
\end{tabular}

Abbreviation: LE, luminous efficacy.

the refractive index and the loading of the phosphor particles, the scattering should be re-evaluated when using other (green) phosphors ${ }^{46}$.

The interplay between both PP and QDs is further demonstrated by the time-resolved photoluminescence. As shown in Figure 4d, mixing them in a single layer leaves the decay of the STG emission unchanged yet substantially raises the QD emission during long delays: $0.5 \mu$ s or longer after photoexcitation. The decay rate of this new long-time component matches that of the STG emission and thus extends the average time constant of the red emission to $369 \mathrm{~ns}$ (see Supplementary Information). We conclude that the QDs in the $\left|R_{A} G_{A}\right|$ layer are excited both directly by the blue pump LED and indirectly by the green light emitted by the STG. Importantly, because the STG emission has an IQE close to unity, this secondary excitation has very little effect on the IQE of the mixed hybrid remote phosphor layer.

Further evidence of the STG emission exciting the QDs comes from an SEM-CL-EDX analysis of a mixed STG/QD layer (Figure 5). An SEM image of the mixed layer shows several STG microcrystals that either protrude from or reside just below the polymer surface, as evidenced by the EDX mapping (Figure $5 \mathrm{c}$ ). The cathodoluminescence (CL) is dominated by the green emission of the STG due to its higher loading, in combination with the low intrinsic CL efficiency for QDs ${ }^{47}$. Subsurface STG crystallites (areas 1 and 2 in Figure 5b) have a longer peak emission wavelength than protruding ones (areas 3 and 4) and feature a weak red emission at wavelengths, where the QD emission is expected. Being absent in STG-only films, these observations cannot, however, be explained as direct CL from QDs in close proximity to subsurface STG crystallites, as this would leave the emission of the latter unchanged. The larger absorption cross-section of the QDs at shorter wavelengths, on the other hand, will make reabsorption of STG emission by the QDs more effective at the short wavelength side of STG emission, explaining the redshifted STG emission. The redshift of the CL emission of the STG, combined with the appearance of the QD emission when exciting subsurface STG crystallites, indicates that QDs indeed reabsorb part of the STG emission.

\section{Stacked two-color QD/PP conversion layers}

We assessed two approaches in the case of two stacked color conversion layers, separating both layers by either an air gap or an index-matching liquid. Comparing first the benchmark $\left|R_{A} G_{A}\right|$ with the $\left|R_{A} \| G_{A}\right|$ and $\left|G_{A} \| R_{A}\right|$ configurations (see Table 2), the emission spectrum strongly depends on the order of the two layers (Figure $4 \mathrm{~b}$ and 4c). When the QD layer is closer to the pump LED $\left(\left|R_{A} \| G_{A}\right|\right)$, the STG emission is strongly suppressed; whereas the QD emission is far more prominent relative to the transmitted blue pump light. By contrast, when the STG layer is closer $\left(\left|G_{A} \| R_{A}\right|\right)$, the emission spectrum is dominated by the green STG band. These changes are explained by Beer-Lambert's law, because the color convertors in the bottom layer are exposed to the highest intensity of blue pump light. 

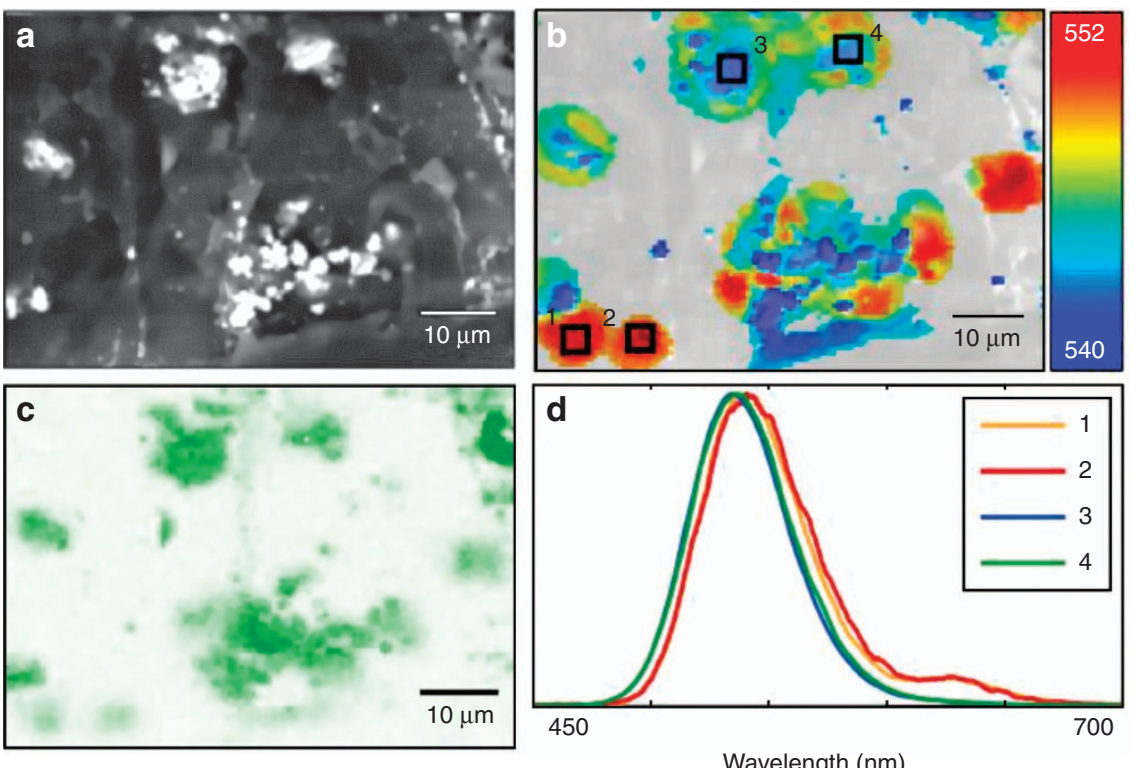

Figure 5 (a) Backscattered electron image of a mixed hybrid layer containing $3.9 \mathrm{~g} \mathrm{~m}^{-2} \mathrm{STG}$ phosphor and $1.0 \mathrm{~g} \mathrm{~m}^{-2} \mathrm{CdSe} / \mathrm{CdS}$ QDs, along with (c) the EDX map for strontium. (b) Map for the barycenter wavelength of the $\mathrm{CL}$ spectra (color scale in $\mathrm{nm}$ ). Areas where the integrated intensity is lower than $1.5 \%$ of the peak intensity are not depicted in color. (d) Emission spectra for the four selected areas indicated in the barycenter map.

The efficiency of the stacked configurations $\left|R_{A} \| G_{A}\right|$ and $\left|G_{A} \| R_{A}\right|$ is mostly affected by the IQE of the respective bottom layer due to the dominance of its emission in the overall spectrum (see Table 2). More specifically, the up to $5.5 \mathrm{~nm}$ redshift of the QD emission indicates that the lower efficiency of $\left|R_{A} \| G_{A}\right|$, as compared with $\left|R_{A} G_{A}\right|$, can be ascribed to the self-absorption of red light trapped in the $\left|R_{A}\right|$ layer, which contains very few scattering centers. Although the $\left|G_{A}\right|\left|R_{A}\right|$ configuration suffers from the same problem, the smaller contribution of the QDs to the emission spectrum reduces its impact on the conversion efficiency. Note that in this configuration, the red emission is partially caused by absorption of the green emission by the QDs, as shown by the additional component in the decay profiles (Figure $4 \mathrm{~d}, \tau_{\mathrm{av}}=455 \mathrm{~ns}$ ). Similar to the mixed layer, reabsorption of the green emission will cause only a slight lowering of the IQE of the wLED.

Compared with $\left|R_{A} \| G_{A}\right|$ and $\left|G_{A} \| R_{A}\right|$, the emission spectra of the index-matched $\left|R_{A} \| G_{A}\right|$ and $\left|G_{A} \| R_{A}\right|$ stacks show an increased red in combination with a decreased green emission intensity, whereas the transmittance of blue pump light and the respective conversion efficiencies remain largely unaffected (Figure $4 \mathrm{~b}$ and $4 \mathrm{c}$ and Table 2). Meanwhile, the QD emission features a markedly reduced redshift of $\lambda_{\max }, 2.5 \mathrm{~nm}$ from $5.5 \mathrm{~nm}$, pointing to a reduced trapping of red light in the $\left|R_{A}\right|$ film. Indeed, the index-matching between the $\left|R_{A}\right|$ and $\left|G_{A}\right|$ layer spreads the red light over the entire stack, where scattering with STG will enhance outcoupling. Moreover, the concomitant lowering of the STG emission and increase of the QD emission—-most pronounced in $\left|G_{A} i R_{A}\right|$ - are evidence of an enhanced reabsorption of STG emission by the QDs. Again, this can be attributed to the index matching, as it allows green light to enter the QD layer under any angle, thus enhancing their average path length in the QD layer. In line with this interpretation, the average lifetime of the QD emission in the $\left|G_{A} \| R_{A}\right|$ configuration increases compared with $\left|G_{A} \| R_{A}\right|$; the traces in Figure $4 c$ show that this indeed reflects a more pronounced secondary excitation by the STG emission.

As the stack geometry boosts or suppresses the contribution of one color convertor to the eventual wLED spectrum, the quantity of each convertor in the respective films must be adjusted to obtain a spectrum with characteristics acceptable for display applications. Meanwhile, the stack geometry offers an opportunity to reduce the material cost of the most expensive compound. We therefore compared the different stack configurations with STG and QD loading tuned to obtain a similar CCT of $6500-7000 \mathrm{~K}$ in all cases. As shown in Figure 6a, approximately similar wLED spectra can be attained for all configurations using the respective amounts of STG and QDs, as indicated in Figure $6 \mathrm{~b}$.

For stacks where the STG is closer to the blue LED, the $\left|G_{B}\right|\left|R_{A}\right|$ configuration can reach a CCT of $\sim 7100 \mathrm{~K}$ with only half the amount of STG as in the mixed $\left|R_{A} G_{A}\right|$ layer, since that emitter is preferentially excited by the incident blue light. Although this configuration suffers from self-absorption of red light by the QDs and the configuration is prone to reabsorption of green light by the QDs, it still has an IQE of $77 \%$ (Table 2). Hence, with the highly efficient STG phosphor and the CdSe/CdS QDs designed to show little self-absorption, reabsorption losses are not a major issue in this hybrid remote phosphor. Interestingly, index-matching both conversion layers yields the configuration $\mid \mathrm{G}_{\mathrm{B}}{ }^{\prime \prime} \mathrm{R}_{\mathrm{C}} \mathrm{l}$, where the QD loading in the remote phosphor has been further reduced by one-third to achieve a wLED spectrum with a CCT of $\approx 6500 \mathrm{~K}$. Clearly, this reduced QD loading reflects the enhanced excitation of the QDs by the STG emission and the improved outcoupling of red light, as discussed above; as a result of these two effects, fewer QDs are needed to produce a similar intensity in the red.

For the $|R \| G|$ configuration, a wLED spectrum comparable to the $\left|R_{A} G_{A}\right|$ benchmark is obtained by incorporating one-third as many QDs and increasing the amount of STG by $20 \%$ in a configuration labeled $\mid R_{B} \| G_{C}$ (Table 2 and Figure 6). Although in this case red light will be trapped in the $\left|R_{B}\right|$ layer, Figure $2 c$ shows that the IQE of a QDonly layer with this specific QD loading is at $80 \%$. This indicates that trapping of the QD emission within the layer does not induce significant self-absorption losses at this lower QD loading for the remote phosphor geometry used here. Thus, we obtain an IQE of 75\% for the entire $\left|R_{B} \| G_{C}\right|$ configuration, markedly higher than the $62 \%$ of 
a

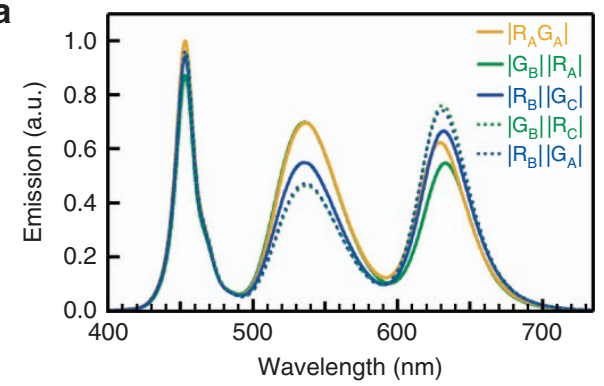

b

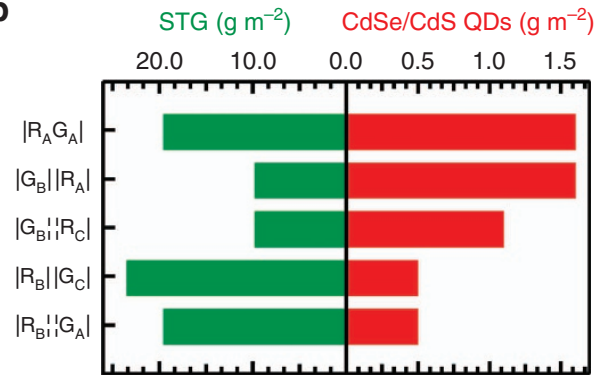

C

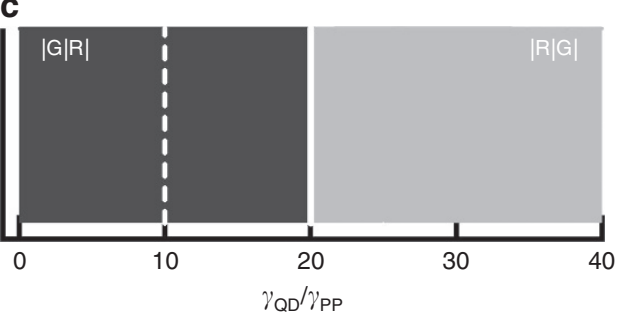

Figure 6 (a) wLED spectra, with a CCT of $6500-7000 \mathrm{~K}$ for all configurations (see Table 2), and (b) loading of STG PP and CdSe/CdS QDs in the layers shown in a. (c) Schematic representation of the design with the lowest cost, depending on the $\gamma_{\mathrm{QD}} / \gamma_{\mathrm{PP}}$ cost ratio.

the previously characterized $\left|\mathrm{R}_{\mathrm{A}} \| \mathrm{G}_{\mathrm{A}}\right|$ stack. In the case of an indexmatched configuration, Figure 6 and Table 2 demonstrate that a CCT of $\sim 6500 \mathrm{~K}$ is obtained for the stack in optical contact, labeled $\left|\mathrm{R}_{\mathrm{B}}{ }_{\mathrm{B}} \mathrm{G}_{\mathrm{A}}\right|$, where the amount of STG could be further reduced compared to $\left|\mathrm{R}_{\mathrm{B}} \| \mathrm{G}_{\mathrm{C}}\right|$.

In concluding the study of the influence of the stacking geometry, one should note that in actual display stacks, different optical layers may be used (such as additional diffusers and prism films), often in combination with strong light recycling. In the present work, light recycling was already partially taken into account by using a white reflective cup. In certain display configurations, the excitation light would pass several times through the STG-QD layer(s), consequently requiring a lower loading of phosphor and quantum dots. Although the influence of the order of PP and QD layers will be reduced, our conclusion regarding the need for scattering to couple out the (red) light remains fully valid. Finally, this hybrid remote phosphor approach could also be used for general lighting applications. The present spectral combination with narrow emission bands, ideal for obtaining a large color gamut in displays, has a typical color rendering index in the mid 70s (for example, color rendering index $=74$ for $\left.\left|R_{A} G_{A}\right|\right)$. To obtain higher color rendering, conversion materials with broader emission bands, or the addition of a third material, should be considered.
Hybrid remote QD/PP design rules

A remote phosphor of interest for display applications is expected to provide the highest performance at the lowest cost. It is reasonable to assume that the cost of the remote phosphor layers is determined primarily by the cost of the color convertor materials such that in the following discussion by 'lowest cost' we mean lowest 'color convertor' cost. Performance, on the other hand, is assessed by considering the properties of the eventual wLED emission spectra and the IQE of the entire color conversion. The proposed hybrid remote QD/PP combination, with CdSe/CdS QDs and STG microcrystals, meets state-ofthe-art spectral specifications for display applications, with an IQE of $75 \%-80 \%$ for all assessed configurations-the latter affected mainly by the somewhat lower IQE of the QDs. This forms an ideal starting point for comparing the configurations by simply considering the cost of the color convertors used.

Expressing the unit cost of QDs and PP as $\gamma_{\mathrm{QD}}$ and $\gamma_{\mathrm{PP}}$ (in cost unit per $\mathrm{g}$ ), respectively, and their respective mass loadings in a remote phosphor layer as $w_{\mathrm{QD}}$ and $w_{\mathrm{PP}}$ (in $\mathrm{g} \mathrm{m}^{-2}$ ), the cost $c$ of the color convertor (in cost unit per $\mathrm{m}^{2}$ ) reads:

$$
c=c_{\mathrm{QD}}+c_{\mathrm{PP}}=\gamma_{\mathrm{QD}} w_{\mathrm{QD}}+\gamma_{\mathrm{PP}} w_{\mathrm{PP}}
$$

Figure $6 \mathrm{~b}$ shows that the least costly designs will be either $\left|\mathrm{R}_{\mathrm{B}}{ }^{11} \mathrm{G}_{\mathrm{A}}\right|$ or $\mid \mathrm{G}_{\mathrm{B}}{ }^{\prime \prime} \mathrm{R}_{\mathrm{C}} \mathrm{l}$, since these minimize $w_{\mathrm{QD}}$ or $w_{\mathrm{PP}}$, respectively. The cost of these configurations will be identical if $\gamma_{\mathrm{QD}} \cong 20 \times \gamma_{\mathrm{PP}}$, taking into account the experimental weights found for both configurations, that is, $w_{\mathrm{PP}} \cong 20 \times w_{\mathrm{QD}}$. This tipping relation is represented as the solid line in the one-dimensional $\gamma_{\mathrm{QD}} / \gamma_{\mathrm{PP}}$ diagram shown in Figure $6 \mathrm{c}$, where it separates cost ratios for which the IR'IGI (light gray area) or the IGIIRI (dark gray area) design is most cost effective. In the dark gray area, the total $\mathrm{PP}$ cost $c_{\mathrm{PP}}$ exceeds the $\mathrm{QD}$ cost $c_{\mathrm{QD}}$, implying that the IGIIRI stack is the more economical of the two. Alternatively, conversion layers can become less expensive in the dark gray region when opting for a QD-only solution, where the green component is also converted by QDs, provided that the same optical efficiency can be achieved. In the light gray area, on the other hand, the QD cost exceeds the PP cost and the IR"IGI design effectively minimizes cost by minimizing the amount of QDs needed. Given their more involved, solution-based synthesis, QDs will generally be more costly than PPs. The cost ratio can then easily exceed the tipping point of $\gamma_{\mathrm{QD}} / \gamma_{\mathrm{PP}} \approx 20$, implying that the cost ratio is found in the light gray area of Figure $6 c$, making the IR"IGI hybrid remote phosphor the most economical choice.

As shown in Figure 6b, shifting either the QDs or the PP from the layer closer to the LED to that farther from it doubles the required weight in the color-converting stack. As long as the color convertor efficiency is close to $100 \%$, this factor of two is universal because it is determined by the fact that identical fractions of the intensity of the blue pump light should be converted to either red or green light in both configurations. Hence, for every system, the tipping ratio, that is, the $\mathrm{QD} / \mathrm{PP}$ cost ratio where both configurations have the same cost, can be expressed as a function of the weight of either convertor:

$$
\frac{\gamma_{\mathrm{QD}}}{\gamma_{\mathrm{PP}}} \approx \frac{w_{\mathrm{PP},|\mathrm{R}:: \mathrm{G}|}}{2 \times w_{\mathrm{QD},|\mathrm{R}:: \mathrm{G}|}}=\frac{w_{\mathrm{QD},|\mathrm{G}:: \mathrm{R}|}}{2 \times w_{\mathrm{PP},|\mathrm{G}:: \mathrm{R}|}}
$$

It thus follows that, in the case of the IR"IGI configuration, for instance, any reduction of the weight of PP needed for the same absorptance of pump light will lower the tipping ratio separating the dark and light gray area in Figure $6 \mathrm{c}$. This is exemplified by the dashed line indicating a $\gamma_{\mathrm{QD}} / \gamma_{\mathrm{PP}}=10$ cost ratio, which represents remote phosphors for which only half the amount of PP as found here would be needed. Such a change can be accomplished by increasing the doping density 
of the STG microcrystals, provided this has no detrimental effect on the QE or the thermal stability of the photoluminescence. Because the operating conditions of remote phosphors in display backlights are less stringent than those for general lighting applications (which operate at higher temperature and flux), the composition and dopant concentration can indeed be optimized. As this will leave $\gamma_{\mathrm{PP}}$ almost unchanged, the lower limit of $\gamma_{\mathrm{QD}}$, where remote $\mathrm{QD} / \mathrm{PP}$ are more cost efficient than QD-only solutions, can thus be further reduced. A similar reduction of the tipping ratio follows from the growth of additional ZnSe or ZnS shells around the QDs-a typical procedure to further enhance their IQE - as these add weight without increasing the absorption coefficient at the pump wavelength. In this respect, it is important to mention that increasing the tipping ratio is less straightforward because with the CdSe/CdS QDs used here, both core and shell fully contribute to the absorption of the pump light and IQEs are already at $75 \%-80 \%$. We thus conclude that a hybrid remote QD/PP will often be a more cost-effective color convertor for display applications than a QD-only combination, for which the larger mass of PP needed is compensated by its reduced cost.

Apart from optimizing cost by minimizing the use of the most expensive color convertor, hybrid remote phosphors can also be used to enhance performance. Here, the most important effect is the suppression of self-absorption of converted light by the QDs in layers where the PP microcrystals and the QDs are either mixed or separated by an index-matching layer. Although self-absorption is less of an issue for the thick shelled CdSe/CdS QDs used here, this is of extreme importance for any QD system in which the shell does not raise the absorption coefficient at the pump wavelength as CdS does. This applies not only to $\mathrm{CdSe} / \mathrm{ZnS}^{48,49}$ or CdSe/CdS QDs with a thin CdS shell but also to typical Cd-free alternatives, such as InP-based QDs and, more recently, perovskite nanocrystals made from cesium lead halide ${ }^{32-34}$. Having comparable absorption coefficients and thus comparable path lengths for blue, green and red light, selfabsorption will increase greatly, as will the efficiency loss, even if the QDs have an IQE of $80 \%-90 \%$. Under these circumstances, a configuration in which QDs and the PP microcrystals are mixed in a single phosphor may be most interesting-even if it requires more material—given the strongest reduction of QD self-absorption in this configuration. Importantly, a similar suppression of self-absorption could be achieved by including low-cost non-luminescent scattering centers in a QD-only remote phosphor coating. This, however, brings us back to a similar cost optimization with a tipping ratio thatassuming that red and green QDs have identical cost and absorption coefficients—can be estimated as:

$$
\frac{\gamma_{\mathrm{QD}}}{\gamma_{\mathrm{PP}}} \approx \frac{w_{\mathrm{PP},|\mathrm{RG}|}}{w_{\mathrm{QD},|\mathrm{RG}|}}
$$

From the data shown in Figure 6b, a tipping ratio of 12.5 is thus calculated for the combination of QDs and STG studied here. Hence, as for QDs where the inclusion of scattering centers overcomes efficiency reduction by self-absorption, hybrid remote QD/PP will often offer a better combination of performance and cost than a QDonly solution because they combine suppression of self-absorption with reduced use of the most expensive color convertor.

\section{CONCLUSION}

We have demonstrated highly efficient hybrid remote conversion layers based on quantum dots and PPs for display backlights by combining green-emitting STG microcrystallites and red emitting CdSe/CdS quantum dots. For a given PP and quantum dot loading, the spectrum and the efficiency of the remote phosphor layer depend on the interplay between both conversion materials, where reabsorption of green emission by the QDs and suppression of self-absorption of red QD emission through enhanced scattering by the PP microcrystals are the main effects. Three different designs were used to fabricate wLEDs with a CCT of 6500-7500 K, a high IQE of 75\%-80\% and a LER exceeding $265 \mathrm{~lm} \mathrm{~W}^{-1}$. The most economical design consists of a layered stack with index-matched spacing between the two convertor layers. We provide general guidelines to reduce the overall cost, based on tipping points for the QD/PP cost ratio. These indicate that a hybrid remote $\mathrm{QD} / \mathrm{PP}$ will often be a more costeffective color convertor for display applications than a QD-only combination.

\section{CONFLICT OF INTEREST}

The authors declare no conflict of interest.

\section{AUTHOR CONTRIBUTIONS}

SA, PFS and ZH conceived the research. SA carried out the synthesis and layer deposition. SA and JJJ performed the characterization and data processing. LIDJM performed and processed the SEM-EDX and cathodoluminescence measurements. SA drafted the manuscript. All authors discussed the results and contributed to the writing of the paper.

\section{ACKNOWLEDGEMENTS}

SA and JJ acknowledge the IWT-Vlaanderen (Agency for Innovation by Science and Technology in Flanders) for a scholarship (111597 and 121024). ZH acknowledges support by the European Commission via the Marie-Sklodowska Curie action Phonsi (H2020-MSCA-ITN-642656), the Belgian Science Policy office (IAP 7.35, photon-ics@be), and Ghent University (GOA 01G01513). PFS thanks the IWT-Vlaanderen for the SBO-IWT grant LumiCoR (SBO130030).

1 Smet PF, Parmentier AB, Poelman D. Selecting conversion phosphors for white lightemitting diodes. J Electrochem Soc 2011; 158: R37-R54.

2 Erdem T, Demir HV. Color science of nanocrystal quantum dots for lighting and displays. Nanophotonics 2013; 2: 57-81.

3 International Telecommunication Union. BT2020-0: Parameter Values for Ultra-High Definition Television Systems for Production and International Programme Exchange. International Telecommunication Union; 2012.

4 Zhu HM, Lin CC, Luo WQ, Shu ST, Liu ZG et al. Highly efficient non-rare-earth red emitting phosphor for warm white light-emitting diodes. Nat Commun 2014; 5: 4312.

5 Pust P, Weiler V, Hecht C, Tücks A, Wochnik AS et al. Narrow-band red-emitting $\mathrm{Sr}\left[\mathrm{LiAl}_{3} \mathrm{~N}_{4}\right]: \mathrm{Eu}^{2+}$ as a next-generation LED-phosphor material. Nat Mater 2014; 13: 891-896.

6 Smyder JA, Krauss TD. Coming attractions for semiconductor quantum dots. Mater Today 2011; 14: 382-387.

7 Wood V, Bulović V. Colloidal quantum dot light-emitting devices. Nano Rev 2010; 1: 5202.

8 Editorial. Nanocrystals in their prime. Nat Nanotechnol 2014; 9: 325.

9 Nizamoglu S, Zengin G, Demir HV. Color-converting combinations of nanocrystal emitters for warm-white light generation with high color rendering index. Appl Phys Lett 2008; 92: 031102

10 Luo ZY, Xu DM, Wu ST. Emerging quantum-dots-enhanced LCDs. J Disp Techno/ 2014; 10: $526-539$

11 Kim TH, Jun S, Cho KS, Choi BL, Jang E. Bright and stable quantum dots and their applications in full-color displays. MRS Bull 2013; 38: 712-720.

12 Chen J, Hardev V, Hartlove J, Hofler J, Lee E. A high-efficiency wide-color-gamut solidstate backlight system for LCDs using quantum dot enhancement film. SID Symp Digest Tech Papers 2012; 43: 895-896.

13 Bourzac K. Quantum dots go on display. Nature 2013; 493: 283.

14 Coe-Sullivan S, Liu WH, Allen P, Steckel JS. Quantum dots for LED downconversion in display applications. ECS J Solid State Sci Technol 2012; 2: R3026-R3030.

15 Jun S, Lee J, Jang E. Highly luminescent and photostable quantum dot-silica monolith and its application to light-emitting diodes. ACS Nano 2013; 7: 1472-1477.

16 Liang RZ, Yan DP, Tian R, Yu XJ, Shi WY et al. Quantum dots-based flexible films and their application as the phosphor in white light-emitting diodes. Chem Mater 2014; 26 : 2595-2600.

17 Wood V, Panzer MJ, Chen JL, Bradley MS, Halpert JE et al. Inkjet-printed quantum dotpolymer composites for full-color AC-driven displays. Adv Mater 2009; 21: 2151-2155.

18 Kong YL, Tamargo IA, Kim H, Johnson BN, Gupta MK et al. 3D printed quantum dot light-emitting diodes. Nano Lett 2014; 14: 7017-7023. 
19 Mutlugun E, Hernandez-Martinez PL, Eroglu C, Coskun Y, Erdem T et al. Large-area (over $50 \mathrm{~cm} \times 50 \mathrm{~cm}$ ) freestanding films of colloidal InP/ZnS quantum dots. Nano Lett 2012; 12: 3986-3993.

20 Cosgun A, Fu RL, Jiang WN, Li JH, Song JZ et al. Flexible quantum dot-PVA composites for white LEDs. J Mater Chem C 2015; 3: 257-264.

21 Shirasaki Y, Supran GJ, Bawendi MG, Bulović V. Emergence of colloidal quantum-dot light-emitting technologies. Nat Photonics 2013; 7: 13-23.

22 Supran GJ, Shirasaki Y, Song KW, Caruge JM, Kazlas PT et al. QLEDs for displays and solid-state lighting. MRS Bull 2013; 38: 703-711.

23 Jang $\mathrm{E}$, Jun S, Jang H, Lim J, Kim B et al. White-light-emitting diodes with quantum dot color converters for display backlights. Adv Mater 2010; 22: 3076-3080.

24 Zhao BX, Zhang DL, Sun K, Wang XB, Mao RH et al. Intrinsic quantum dot based whitelight-emitting diodes with a layered coating structure for reduced reabsorption of multiphase phosphors. RSC Adv 2014; 4: 45155-45158.

25 Chen GH, Yeh CW, Yeh MH, Ho SJ, Chen HS. Wide gamut white light emitting diodes using quantum dot-silicone film protected by atomic layer deposited $\mathrm{TiO}_{2}$ barrier. Chem Commun 2015; 51: 14750-14753.

26 Anc MJ, Pickett NL, Gresty NC, Harris JA, Mishra KC. Progress in non-Cd quantum dot development for lighting applications. ECS J Solid State Sci Technol 2012; 2: R3071-R3082.

27 Li L, Reiss P. One-pot synthesis of highly luminescent InP/ZnS nanocrystals without precursor injection. J Am Chem Soc 2008; 130: 11588-11589.

28 Tessier MD, Dupont D, De Nolf K, De Roo J, Hens Z. Economic and size-tunable synthesis of $\operatorname{InP} / \mathrm{Zn} E(E=\mathrm{S}$, Se) colloidal quantum dots. Chem Mater 2015; 27: 4893-4898.

29 Lee SH, Lee KH, Jo JH, Park B, Kwon Y et al. Remote-type, high-color gamut white light-emitting diode based on InP quantum dot color converters. Opt Mater Exp 2014, 4: 1297-1302.

30 Song WS, Lee SH, Yang H. Fabrication of warm, high CRI white LED using noncadmium quantum dots. Opt Mater Exp 2013; 3: 1468-1473.

$31 \mathrm{Kim} \mathrm{JH}$, Yang $\mathrm{H}$. White lighting device from composite films embedded with hydrophilic $\mathrm{Cu}(\mathrm{In}, \mathrm{Ga}) \mathrm{S}_{2} / \mathrm{ZnS}$ and hydrophobic InP/ZnS quantum dots. Nanotechnology 2014; 25: 225601.

32 Nedelcu G, Protesescu L, Yakunin S, Bodnarchuk MI, Grotevent MJ et al. Fast anionexchange in highly luminescent nanocrystals of cesium lead halide perovskites $\left(\mathrm{CsPb} \mathrm{X}_{3}\right.$, $X=\mathrm{Cl}, \mathrm{Br}, \mathrm{I})$. Nano Lett 2015; 15: 5635-5640.

33 Protesescu L, Yakunin S, Bodnarchuk MI, Krieg F, Caputo R et al. Nanocrystals of cesium lead halide perovskites $\left(\mathrm{CsPb}_{3}, X=\mathrm{Cl}, \mathrm{Br}\right.$, and I): novel optoelectronic materials showing bright emission with wide color gamut. Nano Lett 2015; 15: 3692-3696.

34 Akkerman QA, D'Innocenzo V, Accornero S, Scarpellini A, Petrozza A et al. Tuning the optical properties of cesium lead halide perovskite nanocrystals by anion exchange reactions. J Am Chem Soc 2015; 137: 10276-10281.

35 Chen KJ, Lin CC, Han HV, Lee Cy, Chien SH et al. Wide-range correlated color temperature light generation from resonant cavity hybrid quantum dot lightemitting diodes. IEEE J Select Topics Quantum Electron 2015; 21: 1900407.
36 Hirayama R, Naruse M, Nakayama H, Tate N, Shiraki A et al. Design, implementation and characterization of a quantum-dot-based volumetric display. Sci Rep 2015; 5: 8472.

37 Carbone L, Nobile C, De Giorgi M, Sala FD, Morello G et al. Synthesis and micrometerscale assembly of colloidal CdSe/CdS nanorods prepared by a seeded growth approach. Nano Lett 2007; 7: 2942-2950.

38 Jasieniak J, Smith L, van Embden J, Mulvaney P, Califano M. Re-examination of the size-dependent absorption properties of CdSe quantum dots. J Phys Chem C 2009; 113: 19468-19474.

39 Cirillo M, Aubert T, Gomes R, van Deun R, Emplit P et al. "Flash" synthesis of CdSe/CdS core-shell quantum dots. Chem Mater 2014; 26: 1154-1160.

40 Leyre S, Coutino-Gonzalez E, Joos JJ, Ryckaert J, Meuret Y et al. Absolute determination of photoluminescence quantum efficiency using an integrating sphere setup. Rev Sci Instrum 2014; 85: 123115.

41 Davis W, Ohno Y. Toward an Improved Color Rendering Metric. Proc SPIE 2005; 5941: $59411 \mathrm{G}$.

42 Ohno Y. Color Rendering and Luminous Efficacy of White LED Spectra. Proc SPIE 2004; 5530: 88-98.

43 Ohno Y. Spectral design considerations for white LED color rendering. Opt Eng 2005; 44: 111302.

44 Poelman D, Smet PF. Time resolved microscopic cathodoluminescence spectroscopy for phosphor research. Phys B 2014; 439: 35-40.

45 Woo JY, Kim K, Jeong S, Han CS. Enhanced photoluminance of layered quantum dotphosphor nanocomposites as converting materials for light emitting diodes. J Phys Chem C 2011; 115: 20945-20952.

46 Park HK, Oh JH, Do YR. Toward scatter-free phosphors in white phosphor-converted light-emitting diodes. Opt Exp 2012; 20: 10218-10228.

47 Fern GR, Silver J, Coe-Sullivan S. Cathodoluminescence and electron microscopy of red quantum dots used for display applications. J Soc Inf Disp 2015; 23: 50-55.

48 Čapek RK, Lambert K, Dorfs D, Smet PF, Poelman D et al. Synthesis of extremely small $\mathrm{CdSe}$ and bright blue luminescent $\mathrm{CdSe} / \mathrm{ZnS}$ nanoparticles by a prefocused hotinjection approach. Chem Mater 2009; 21: 1743-1749.

49 Nizamoglu S, Demir HV. Excitation resolved color conversion of CdSe/ZnS core/shell quantum dot solids for hybrid white light emitting diodes. J Appl Phys 2009; 105 083112.

(c) (i) (2) This work is licensed under a Creative Commons AttributionBY NC SA NonCommercial-ShareAlike 4.0 International License. The images or other third party material in this article are included in the article's Creative Commons license, unless indicated otherwise in the credit line; if the material is not included under the Creative Commons license, users will need to obtain permission from the license holder to reproduce the material. To view a copy of this license, visit http:// creativecommons.org/licenses/by-nc-sa/4.0/

(C) The Author(s) 2017

Supplementary Information for this article can be found on the Light: Science \& Applications' website (http://www.nature.com/lsa). 